This seems to be the generalization of the classical result that a necessary and sufficient condition for the polar components of a matrix $A$ to be commutative is that $A$ be a normal matrix.

Queens College

\title{
REMARKS ON REGULARITY OF METHODS OF SUMMATION
}

\section{G. E. FORSYTHE AND A. C. SCHAEFFER}

A doubly infinite matrix ${ }^{1}\left(a_{m n}\right)(m, n=1,2, \cdots)$ is said to be regu$l a r$, if for every sequence $x=\left\{x_{n}\right\}$ with limit $x^{\prime}$ the corresponding sums $y_{m}=\sum_{n=1}^{\infty} a_{m n} x_{n}$ exist for $m=1,2, \cdots$, and if $\lim _{m \rightarrow \infty} y_{m}=x^{\prime}$. An apparently more inclusive definition of regularity is that for each sequence $x$ with limit $x^{\prime}$ the sums defining $y_{m}$ shall exist for all $m \geqq m_{0}(x)$ and $\lim _{m \rightarrow \infty} y_{m}=x^{\prime}$. Tamarkin 2 has shown that $\left(a_{m n}\right)$ is regular in the latter sense if and only if there exists an $m_{1}$ independent of $x$ such that the matrix $\left(a_{m n}\right)\left(m \geqq m_{1}, n \geqq 1\right)$ is regular in the former sense. Using point set theory in the Banach space $(c)$, he proves a theorem ${ }^{3}$ from which follows the result just mentioned. This note presents an elementary proof of that theorem and discusses some related topics.

THEOREM 1. Suppose the doubly infinite matrix $\left(a_{m n}\right)$ has the property that for each sequence $x=\left\{x_{n}\right\}$ with limit 0 there exists an $m_{0}=m_{0}(x)$ such that for all $m \geqq m_{0}(x), u_{m}=\lim \sup _{k \rightarrow \infty}\left|\sum_{n=1}^{k} a_{m n} x_{n}\right|<\infty$. Then there exists an $m_{1}$ such that $\sum_{n=1}^{\infty}\left|a_{m n}\right|<\infty$ for all $m \geqq m_{1}$.

If in addition $\lim _{m \rightarrow \infty} u_{m}=0$ for each sequence $x$ with limit 0 , it will follow ${ }^{4}$ that there exists an $N$ such that $\sum_{n=1}^{\infty}\left|a_{m n}\right| \leqq N<\infty$, for all $m \geqq m_{1}$.

To prove Theorem 1, suppose there were an infinite sequence $m_{1}<m_{2}<\cdots$ such that $\sum_{n=1}^{\infty}\left|a_{m n}\right|=\infty$ for $m \in\left\{m_{\nu}\right\}$. Let $x_{1}, \cdots, x_{k_{1}}$ be chosen with unit moduli and with amplitudes such that

Presented to the Society, April 11, 1942 under the title $A$ remark on Toeplitz matrices; received by the editors January 22, 1942.

${ }^{1}$ In this note $a_{m n}, x_{n}$ and $x^{\prime}$ denote finite complex numbers.

${ }^{2} \mathrm{~J}$. D. Tamarkin, On the notion of regularity of methods of summation of infinite series, this Bulletin, vol. 41 (1935), pp. 241-243.

${ }^{3}$ J. D. Tamarkin, loc. cit., p. 242, lines 1-6.

${ }^{4}$ See, for example, I. Schur, Über lineare Transformationen in der Theorie der unendlichen Reihen, Journal für die reine und angewandte Mathematik, vol. 151 (1921), pp. 79-111; p. 85, Theorem 4. 


$$
\sum_{n=1}^{k_{1}} a_{m_{1} n} x_{n}=\sum_{n=1}^{k_{1}}\left|a_{m_{1} n} x_{n}\right|>1 .
$$

Let $x_{k_{1}+1}, \cdots, x_{k_{2}}$ be chosen with moduli $1 / 2$ and with amplitudes such that

$$
\left|\sum_{n=k_{1}+1}^{k_{2}} a_{m_{2} n} x_{n}\right|>2+\sum_{n=1}^{k_{1}}\left|a_{m_{2} n} x_{n}\right| .
$$

Let $x_{k_{2}+1}, \cdots, x_{k_{3}}$ be chosen with moduli $1 / 3$ and with amplitudes such that

$$
\left|\sum_{n=k_{2}+1}^{k_{3}} a_{m_{1} n} x_{n}\right|>3+\sum_{n=1}^{k_{2}}\left|a_{m_{1} n} x_{n}\right| .
$$

Writing $y_{m}(k)=\sum_{n=1}^{k} a_{m n} x_{n}$, the sequence $\left\{x_{n}\right\}$ and integers $k_{1}<k_{2}<\ldots$ are thus chosen successively so that $\left|y_{m_{1}}\left(k_{1}\right)\right|>1$, $\left|y_{m_{2}}\left(k_{2}\right)\right|>2 ;\left|y_{m_{1}}\left(k_{3}\right)\right|>3,\left|y_{m_{2}}\left(k_{4}\right)\right|>4,\left|y_{m_{3}}\left(k_{5}\right)\right|>5 ;\left|y_{m_{1}}\left(k_{6}\right)\right|>6$, ... ; while $\left|x_{n}\right|=1 / r$, for $k_{r-1}<n \leqq k_{r}$. This is a sort of alternating or "sweeping-out" process. So defined, $\left\{x_{n}\right\}$ is a sequence with limit 0 , but $\lim \sup _{k \rightarrow \infty}\left|\sum_{n=1}^{k} a_{m n} x_{n}\right|=\infty$, for $m \in\left\{m_{\nu}\right\}$. This contradiction completes the proof of Theorem 1.

The matrix $\left(a_{m n}\right)$ is said to be null-preserving, if for every sequence $x=\left\{x_{n}\right\}$ with limit 0 the corresponding sums defining $y_{m}$ exist for $m=1,2, \cdots$ and if $\lim _{m \rightarrow \infty} y_{m}=0$. An apparently more inclusive definition of null-preserving is that for each sequence $x$ with limit 0 we have $u_{m}=\lim \sup _{k \rightarrow \infty}\left|\sum_{n=1}^{k} a_{m n} x_{n}\right|<\infty$ for all $m \geqq m_{0}(x)$ and $\lim _{m \rightarrow \infty} u_{m}=0$. We remark that it is a consequence of Theorem 1 that $\left(a_{m n}\right)$ is null-preserving in the latter sense if and only if there exists an $m_{1}$ such that the matrix $\left(a_{m n}\right)\left(m \geqq m_{1}, n \geqq 1\right)$ is null-preserving in the former sense. ${ }^{5}$

To consider a problem which is related to the above in the method of proof, let each element of a matrix $\left(a_{m n}\right)$ be either +1 or -1 . For $0 \leqq t \leqq 1$ and $n=1,2, \cdots$ let $\left\{\phi_{n}(t)\right\}$ be the Rademacher orthogonal functions, ${ }^{6}$ and let $y_{m k}(t)=\sum_{n=1}^{k} a_{m n} \phi_{n}(t)$. Then it is well known ${ }^{7}$ that for almost all $t$, for all $m=1,2, \cdots$ and for all $\epsilon>0, \lim _{k \rightarrow \infty} k^{-1 / 2-\epsilon} y_{m k}(t)$ $=0$. It is clear that for a particular fixed $m$ there is a $t$ such that $\lim _{k \rightarrow \infty} k^{-1} y_{m k}(t)=1$. The problem is to show that there is a $t$ such that

${ }^{5}$ For conditions that $\left(a_{m n}\right)$ be null-preserving, see T. Kojima, On generalized Toeplitz's theorems on limit and their applications, Tôhoku Mathematical Journal, vol. 12 (1917), pp. 291-326; p. 300.

${ }^{6}$ A. Zygmund, Trigonometrical Series, Warsaw, 1935, p. 5.

${ }^{7}$ For references to this and more precise results, see A. Khintchine, Asymptotische Gesetze der Wahrscheinlichkeitsrechnung, Ergebnisse der Mathematik, Berlin, 1933, pp. 60-61. 
simultaneously for all $m=1,2, \cdots, \lim \sup _{k \rightarrow \infty} k^{-1} y_{m k}(t)=1$. That there exists such a $t$ can be shown by using the alternating process of Theorem 1.

Theorem 2 follows immediately from a theorem of Banach. ${ }^{8}$

THEOREM 2. If $E_{m}$ is a linear manifold satisfying Baire's condition ${ }^{9}$ in a Banach space $E(m=1,2, \ldots)$ and if $\lim _{m \rightarrow \infty} E_{m}=E$, then there exists an $m_{1}$ such that $E_{m_{1}}=E$.

Theorem 2 furnishes a Banach space analogue and a proof of Theorem 1 which is related to Tamarkin's proof. To see this, let $E$ be the Banach space $\left(c_{0}\right)$ of sequences $x=\left\{x_{n}\right\}$ convergent to 0 , with $\|x\|=\max _{n}\left|x_{n}\right|$, and with addition and multiplication by a (complex) scalar defined as usual. Let $\left(a_{m n}\right)$ be as in Theorem 1. Let $E_{m}$ be the subset of $E$ for which $\lim \sup _{k \rightarrow \infty}\left|\sum_{n=1}^{k} a_{r n} x_{n}\right|<\infty$ for all $r \geqq m$. The hypotheses of Theorem 2 are satisfied, and from its conclusion it may be proved directly for an arbitrary $m \geqq m_{1}$ that $\sum_{n=1}^{\infty}\left|a_{m n}\right|<\infty$.

\section{STANFORd UNIVERSity}

${ }^{8}$ S. Banach, Théorie des Opérations Linéaires, Warsaw, 1932, p. 22, Theorem 2.

${ }^{9}$ See S. Banach, loc. cit., p. 17. By considering a Hamel base for $E$, G. W. Mackey has remarked to the authors that Theorem 2 is false if the words "satisfying Baire's condition" are omitted. 